\title{
Lower Gastrointestinal Hemorrhage
}

National Cancer Institute

\section{Source}

National Cancer Institute. Lower Gastrointestinal Hemorrhage. NCI Thesaurus. Code C78438.

Bleeding from the lower gastrointestinal tract (small intestine, large intestine, and anus). 